\title{
Comparison of the so-called CGR and NCR cathodes in commercial lithium-ion batteries using in situ neutron powder diffraction
}

\author{
Moshiul Alam, ${ }^{1,2}$ Tracey Hanley, ${ }^{2}$ Wei Kong Pang, ${ }^{2,3}$ Vanessa K. Peterson, ${ }^{2}$ and Neeraj Sharma ${ }^{1, a)}$ \\ ${ }^{I}$ School of Chemistry, UNSW Australia, Sydney, NSW 2052, Australia \\ ${ }^{2}$ Australian Nuclear Science and Technology Organisation, Kirrawee DC, NSW 2232, Australia \\ ${ }^{3}$ Faculty of Engineering, School of Mechanical, Materials, and Mechatronic Engineering, Institute for \\ Superconducting and Electronic Materials, University of Wollongong, NSW 2522, Australia
}

(Received 2 September 2014; accepted 30 September 2014)

\begin{abstract}
The evolution of the 003 reflection of the layered $\mathrm{Li}(\mathrm{Ni}, \mathrm{Co}, \mathrm{Mn}) \mathrm{O}_{2}(\mathrm{CGR})$ and $\mathrm{Li}(\mathrm{Ni}, \mathrm{Co}, \mathrm{Al}) \mathrm{O}_{2}(\mathrm{NCR})$ cathodes in commercial 18650 lithium-ion batteries during charge/discharge were determined using in situ neutron powder diffraction. The 003 reflection is chosen as it is the stacking axis of the layered structure and shows the largest change during charge/discharge. The comparison between these two cathodes shows that the NCR cathode exhibits an unusual contraction near the charged state and during the potentiostatic step, where the potentiostatic step is recommended by the manufacturer. This feature is not shown to the same degree by the CGR cathode. The behavior is likely related to the compositions of these cathodes, the amount of $\mathrm{Li} / \mathrm{Ni}$ site mixing and the presence of $\mathrm{Al}$ or $\mathrm{Mn}$. (C2014 International Centre for Diffraction Data. [doi:10.1017/S088571561400102X]
\end{abstract}

Key words: single-peak fitting, time-resolved in situ diffraction

\section{INTRODUCTION}

Lithium-ion batteries are found in many portable applications, providing stored energy for use. Although lithium-ion batteries meet the majority of the demands put on them by current applications, emerging applications such as electric vehicles and grid-scale energy storage requires improvements in aspects such as safety, cost, energy density, and high-current performance (Tarascon and Armand, 2001; Goodenough and Kim, 2011). The cathode plays an essential role in the performance of a lithium-ion battery and often is the limiting factor in performance parameters. The major reason the cathode is the limiting factor is because it is the lithium source and its capacity (reversible lithium content) is lower than that provided by the widely used graphite anode. The cathode has been continuously improved by researchers and industry to deliver the above-mentioned improvements.

The first commercialized lithium-ion battery by SONY in 1991 (Nagaura and Tozawa, 1990), consisted of a $\mathrm{LiCoO}_{2}$ cathode with a graphite anode. Recently new cathode chemistries have been developed and commercialized both for specialized applications such as electric vehicles and to replace the more widespread use of $\mathrm{LiCoO}_{2}$ cathodes, for example $\mathrm{LiFePO}_{4}$ (Padhi et al., 1997) and $\mathrm{LiMn}_{2} \mathrm{O}_{4}$ (Thackeray et al., 1992). Notably, a number of cathodes adopting a similar layered type $R \overline{3} \mathrm{~m}$ structure are found to alleviate some of the issues with the $\mathrm{LiCoO}_{2}$ cathode. These cathodes include $\mathrm{LiNi}_{1 / 3} \mathrm{Co}_{1 / 3} \mathrm{Mn}_{1 / 3} \mathrm{O}_{2}$ with varying ratios of $\mathrm{Li}: \mathrm{Ni}: \mathrm{Co}: \mathrm{Mn}$ and $\mathrm{Al}$-doped $\mathrm{Li}(\mathrm{NiCo}) \mathrm{O}_{2}$ (Dolotko et al., 2014). Reduction in the Co content of these cathodes reduces cost and the environmental impact as Co is a toxic element. This is particularly true if similar or superior electrochemical performance can be

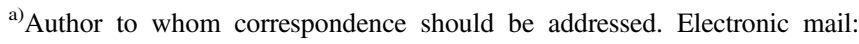
neeraj.sharma@unsw.edu.au
}

delivered. Although the starting cathodes have been thoroughly characterized the question remains: what does the inclusion of $\mathrm{Ni}, \mathrm{Co}, \mathrm{Mn}$, and $\mathrm{Al}$ do to the structural evolution as a function of charge/discharge? For example, do the cathodes show continuous expansion/contraction during charge/discharge or do they differ?

A non-destructive method to probe cathode structural evolution in commercial 18650 or alternate-shaped batteries is time-resolved in situ neutron powder diffraction (NPD). Early studies focused on essentially in situ NPD patterns collected over extended periods of time with batteries equilibrated at a particular state of charge (Rodriguez et al., 2004; Senyshyn et al., 2012). Recent work has taken advantage of the improvements in neutron sources and detectors to undertake time-resolved measurements particularly on $\mathrm{LiCoO}_{2}$ llgraphite batteries, revealing the presence of new phases during charge/discharge, for example spinel $\mathrm{LiCoO}_{2}$ (Sharma et al., 2010; Sharma and Peterson, 2012), evaluating the time-dependent nature of structural evolution (Wang et al., 2012), and determining the influence of overcharging on the structure and electrochemical properties of the electrodes (Sharma and Peterson, 2013b). Thus, time-resolved in situ NPD is a powerful tool to study the evolution of electrodes in commercial lithium-ion batteries.

Recently, an NPD study investigated cation-site mixing in $\mathrm{Li}\left(\mathrm{Ni}_{0.5} \mathrm{Mn}_{0.3} \mathrm{Co}_{0.2}\right) \mathrm{O}_{2}$ cathodes in 18650 batteries with in situ NPD using collection times of 30 min per pattern and analyzed by single-peak fitting of the cathode 003 and 217 reflections. The structural evolution was analyzed using Rietveld analysis of equilibrated cathodes collected for $4 \mathrm{~h}$ at a desired state of charge of the battery. This work, based on data for the cathode equilibrated at a particular state of charge, illustrated a change in the lattice parameter trend during charge. The volume contracts for most of charge and contracts at a faster rate near completion and this was related to the 
cation mixing in the cathode (Dolotko et al., 2014). This motivated our work where we focus here on a comparison of the evolution of the 003 reflections of the $\mathrm{Li}(\mathrm{Ni}, \mathrm{Co}, \mathrm{Mn}) \mathrm{O}_{2}$ (CGR) and $\mathrm{Li}(\mathrm{Ni}, \mathrm{Co}, \mathrm{Al}) \mathrm{O}_{2}(\mathrm{NCR})$ electrodes during a similar charge/ discharge cycle to illustrate the anomaly near the charged state. The 003 reflection is the stacking axis of the layered structure and is expected to show the largest change during charge/discharge. By comparing the above-mentioned two commercially available electrodes, this work details the different evolution and provides rationale for their performance.

\section{EXPERIMENTAL}

Commercial lithium-ion batteries (18 650) were obtained commercially. The batteries were denoted as CGR and NCR, which corresponded to the compositions CGR and NCR, respectively. These batteries are sold at a state of charge ranging from 50 to $60 \%$. In situ NPD data were collected on WOMBAT (Studer et al., 2006), the high-intensity powder diffractometer, at the Open Pool Australian Light-water (OPAL) reactor facility at the Australian Nuclear Science and Technology Organisation (ANSTO), as per our previous work (Sharma et al., 2010, 2011a, 2011b, 2012, 2013; Sharma and Peterson, 2012, 2013a, 2013b; Hu et al., 2013; Pang et al., 2014). The batteries were placed in a neutron beam of wavelength 2.4105(3) $\AA$, determined using the $\mathrm{Al}_{2} \mathrm{O}_{3}$ NIST SRM 676, and data collected in the range $24^{\circ} \leq 2 \theta \leq 144$. NPD data were acquired every 5 min during charge/discharge cycling. NPD data correction, reduction, and visualization were undertaken using the program LAMP (Richard et al., 1996). Rietveld analyses were carried out using the GSAS (Larson and Von Dreele, 1994) suite of programs with the EXPGUI (Toby, 2001) interface.

During the in situ NPD experiment the electrochemical cell was cycled in galvanostatic-potentiostatic mode as recommended by the manufacturer between 2.5 and $4.2 \mathrm{~V}$ using an Autolab potentiostat/galvanostat (PG302N) under ambient conditions, with the external temperature maintained at $22.5^{\circ} \mathrm{C}$. Cycles were performed with varying current from 0.5 to $2 \mathrm{~A}$. Information regarding the battery composition, performance, and details are derived from the information sheet provided by the manufacturer. These batteries are rated to deliver 2.25 Ah (CGR) and 3.1 Ah (NCR) using constant current charging until $4.2 \mathrm{~V}$ and holding at $4.2 \mathrm{~V}$ using a tapering current (potentiostatic step).

\section{RESULTS AND DISCUSSION}

The CGR cathode composition is quoted as $\mathrm{LiNi}_{1 / 3} \mathrm{Co}_{1 / 3}$ $\mathrm{Mn}_{1 / 3} \mathrm{O}_{2}$ in some commercial datasheets but often referred to as CGR, whereas the NCR cathode composition is only given as a mixture of $\mathrm{Ni}, \mathrm{Co}$, and $\mathrm{Al}$ where the $\mathrm{Al}$ dopant is used to optimize performance. The as-collected NPD patterns for the as-obtained batteries are shown in [Figure 1(a)], and we note a significant background contribution from the hydrogen containing components in the battery [see Sharma et al. (2011a) for more details]. The striking feature of these patterns is the similarity of the diffraction patterns in terms of peak positions of both batteries. This arises because the batteries are effectively the same construction (in terms of materials and composition) and are at an approximately equivalent state of charge with the only difference being the cathode composition.
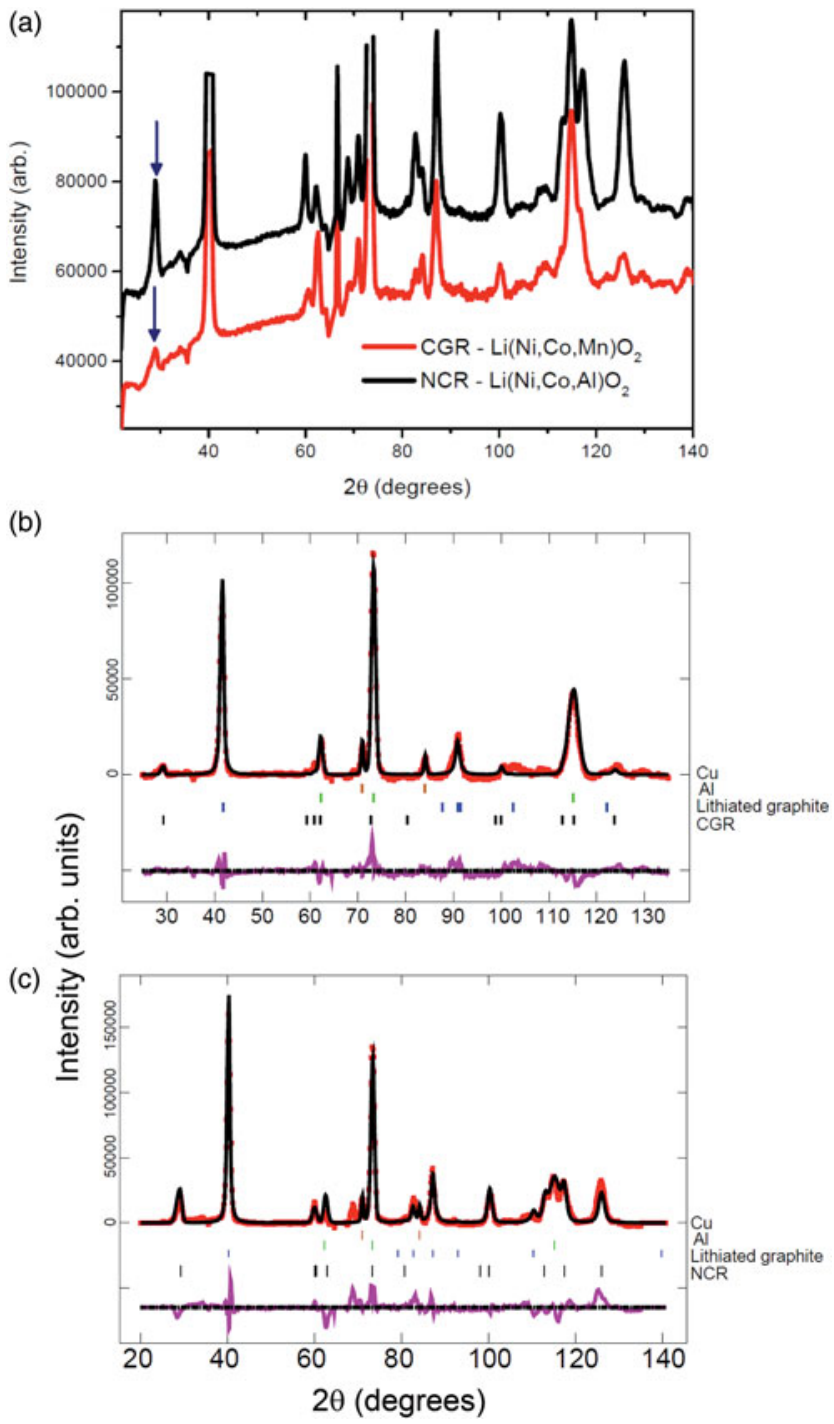

Figure 1. (Color online) (a) The as-collected diffraction patterns of the NCR and CGR containing batteries. The reflection at $\sim 41^{\circ} 2 \theta$ has been truncated for ease of comparison. The Rietveld refined fits of structural models to the first in situ NPD data with the (b) CGR and (c) NCR battery. Data are shown as crosses, the calculated Rietveld model as a line through the data, and the difference between the data and the model as the line below the data. The vertical reflection markers are for copper (brown) and aluminum (green) current collectors, lithiated graphite anode (blue) and the respective cathodes (black).

To model these in situ patterns the $\mathrm{LiNi}_{1 / 3} \mathrm{Co}_{1 / 3} \mathrm{Mn}_{1 / 3} \mathrm{O}_{2}$ model from Dolotko et al. (2014) is used, and the refined lattice parameters are $a=2.855(2)$ and $c=14.28(2) \AA$ for the first dataset of the CGR battery. The same model was used for the NCR battery by adjusting for composition, removing $\mathrm{Mn}$ and placing an initial guess of $5 \% \mathrm{Al}$ on the transition metal (TM) site, resulting in the lattice parameters $a=2.8264(5)$ and $c=14.367(5) \AA$. The NCR lattice is slightly larger in the $c$-stacking axis but smaller in the $a b$-plane compared with CGR. The fits are shown in [Figures 1(b) and 1(c)]; it should be noted that these are highly constrained Rietveld refinements as the atomic and profile parameters are refined and fixed, and only the lattice parameters continuously refined. The arrow in [Figure 1(a)] shows the cathode 003 reflection, which between the NCR and CGR datasets changes in intensity, while the position remains effectively the same. 


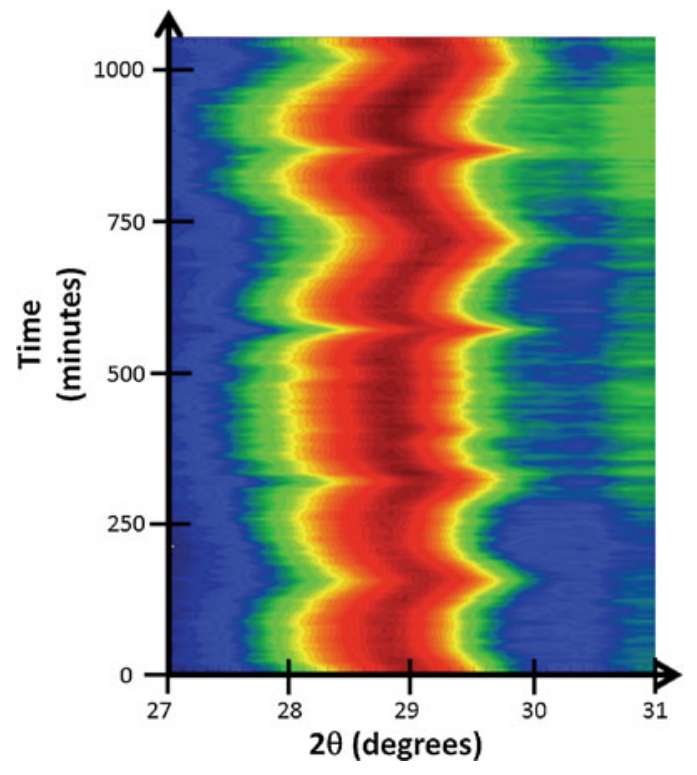

Figure 2. (Color online) 2D color plot showing the evolution of the 003 reflection of NCR during charge/discharge in the in situ NPD experiment.

Highly constrained Rietveld analyses were performed to identify the remaining battery components, and five components were identified using the starting structural models of the cathode(s), graphite, $\mathrm{LiC}_{6}, \mathrm{Cu}$, and $\mathrm{Al}$ [Figures 1(b) and 1(c)]. The cathode adopts $R \overline{3} \mathrm{~m}$ symmetry with Li layers and layers of $M \mathrm{O}_{6}$, where $M=\mathrm{Ni}, \mathrm{Co}, \mathrm{Mn}$, and $\mathrm{Al}$ and in some instances the $M \mathrm{O}_{6}$ layers can show some long-range ordering of the cations and/or contain $\mathrm{Li}^{+}$(Dolotko et al., 2014). In the case of $\mathrm{Li}\left(\mathrm{Ni}_{0.5} \mathrm{Mn}_{0.3} \mathrm{Co}_{0.2}\right) \mathrm{O}_{2}$, detailed structural analysis reveals $\mathrm{Ni}$ in the $\mathrm{Li}$ layers ( $3 a$ site) and $\mathrm{Li}$ in the $\mathrm{MO}_{6}$ layers ( $3 b$ site) and this interchange is in part facilitated by the similar ionic radii of the $\mathrm{Li}$ and $\mathrm{Ni}$ cations (Dolotko et al., 2014). This work shows that lithium is extracted predominantly from the $3 a$ sites and that the TM $3 b$ site contains lithium until at least $\sim 70 \%$ state of charge of the battery, after which lithium is no longer present at the TM $3 b$ site. Notably the removal of lithium from the TM $3 b$ site occurs close to a large contraction of the $c$-lattice parameter during charge (Dolotko et al., 2014), opposing the trend during charge.

The evolution of the 003 reflection of the cathodes is shown in Figure 2 illustrating changes in the $2 \theta$ value during charge/discharge. Multiple charge/discharge cycles are shown in Figure 2, and the 003 reflection over the course of cycling was fitted with a single Lorentzian peak. The CGR 003 and NCR 003 reflection position, integrated intensity, and fullwidth at half-maximum (FWHM) are shown in Figures 3 and 4 , respectively in a comparable full-charge/discharge cycle of these batteries.

If we consider the CGR cathode first, then there is a gradual increase in integrated intensity and decrease in FWHM during charge (Figure 3). Although the reflection position shows a decreasing trend for most of charge and stabilizes near the charged state and remains at this value during the potentiostatic step and the initial stages of discharge. During discharge all three fit parameters show the reverse behavior. The stabilized region during the potentiostatic step does show a slight decrease in the 003 reflection position similar to that reported for a potentiostatic hold step for $\mathrm{Li}_{1.2} \mathrm{Co}_{0.1} \mathrm{Mn}_{0.55} \mathrm{Ni}_{0.15} \mathrm{O}_{2}$ (Mohanty et al., 2013) and in $\mathrm{Li}\left(\mathrm{Ni}_{0.5} \mathrm{Mn}_{0.3} \mathrm{Co}_{0.2}\right) \mathrm{O}_{2}$ (Dolotko et al., 2014). However, the response or stabilization in the CGR battery studied here shows a less dramatic or smaller change near the charged state relative to the larger contraction near the charged state in $\mathrm{Li}\left(\mathrm{Ni}_{0.5} \mathrm{Mn}_{0.3} \mathrm{Co}_{0.2}\right) \mathrm{O}_{2}$ (Dolotko et al., 2014). This may be related to our work probing more of a kinetic response in the cathode because of the shorter collection times, 5 relative to 30 or $240 \mathrm{~min}$, or differences in cathode composition.

Comparing the NCR cathode with the CGR cathode, the major difference in behavior is found near the charged state, where the 003 reflection position changes from a continuously decreasing trend during charge, reversing in sign and increasing during the potentiostatic step. On initiation of the discharge step the reflection position decreases until it reaches a minimum position and then increases for the reminder of discharge. The behavior of the integrated intensity and FWHM is also different in the NCR relative to CGR cathode, with maximum FWHM and minimum integrated intensity observed at the charged and discharged states.

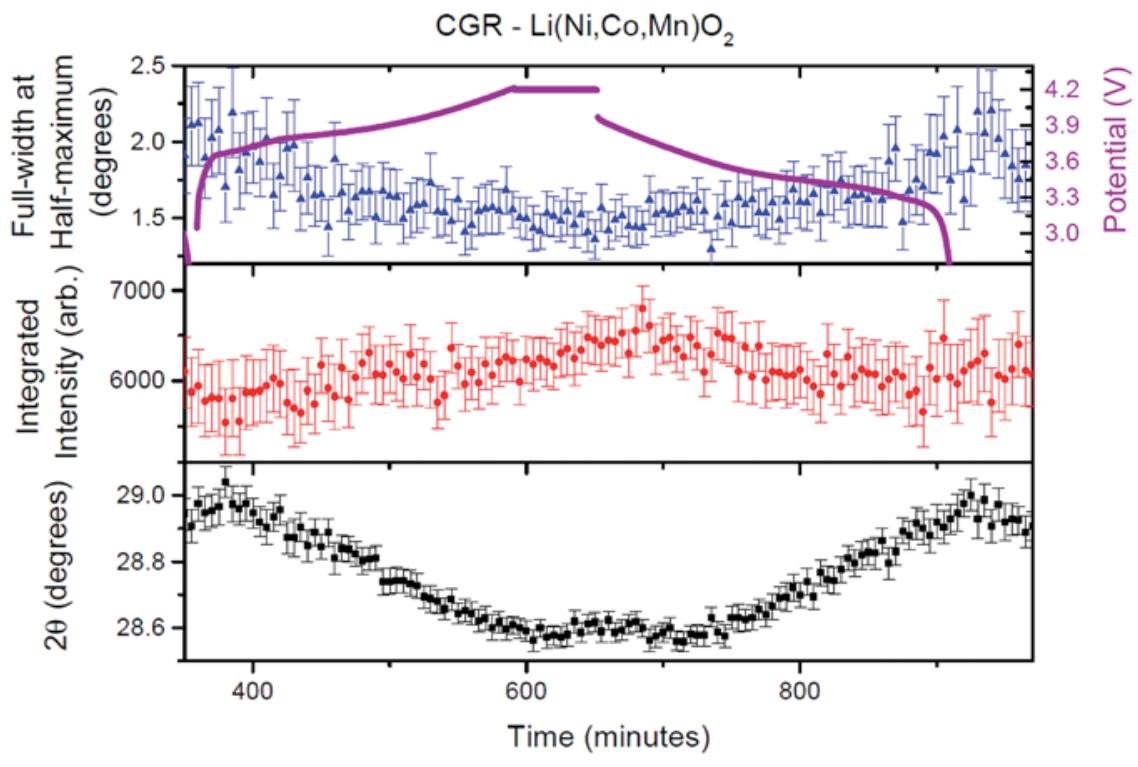

Figure 3. (Color online) The CGR 003 reflection position, integrated intensity, and FWHM during one charge/discharge cycle. The potential profile is also shown. 


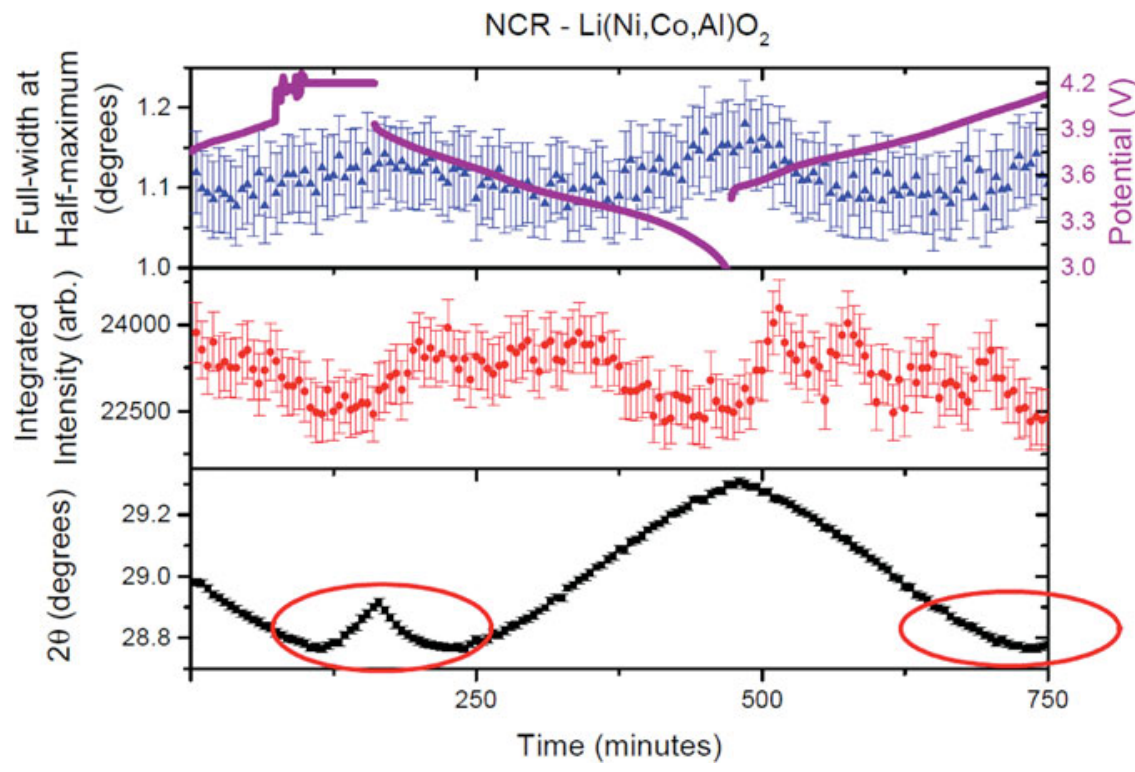

Figure 4. (Color online) The NCR 003 reflection position, integrated intensity, and FWHM during one charge/discharge cycle. The potential profile is also shown.

The 003 reflection is indicative of the changes in the $c$-lattice parameter, representing the stacking axis of the layered structure. During charge the decrease in the reflection position, corresponding to an increase in the $c$-lattice parameter, is a consequence of the lithium extraction from the lithium layers and the repulsion of the overall negatively charged TM containing octahedral layers, for example $T M^{2+} \mathrm{O}_{6}^{-4}$ to $T M^{4+}$ $\mathrm{O}_{6}^{-2}$ (Sharma et al., 2010). This accounts for the capacity of the battery during this period. Near the charged state and the potentiostatic step the stabilization for CGR and decrease in $c$-lattice parameter for NCR batteries suggests an alternative mechanism is occurring to give rise to capacity. This stabilization and/or decrease in the $c$-lattice parameter are likely to be related to the removal of $\mathrm{Li}$ from the TM layer in these cathodes as proposed (Dolotko et al., 2014). It is interesting to note the differences in the magnitude of change near the charged state, where NCR shows a contraction in the lattice, whereas CGR shows stabilization. The differences in these cathodes relate to the quantity of $\mathrm{Ni}$ and $\mathrm{Co}$ and therefore to the relative amount of $\mathrm{Ni}$ on the $\mathrm{Li}$ layers and $\mathrm{Li}$ in the $\mathrm{TM}$ (Ni) layers, and the presence of $\mathrm{Mn}$ or $\mathrm{Al}$. In order for the TM layers to come closer (contract as observed in NCR), the overall negative charge on the TM layers must be counteracted by an attractive component between the layers or a reduction of the overall negative charge. Speculatively this may be because of $\mathrm{Li}^{+}$in the TM layer exchanging for $\mathrm{Ni}^{2+}$ in the lithium layer, reducing the overall negative charge of the TM layer and leading to a contraction. Although alternate explanations are possible, future and modified in situ neutron diffraction experiments (for example, extending the potentiostatic step) are underway to determine the precise origin of these differences.

\section{CONCLUSION}

Here we illustrate how in situ NPD allows comparison of the structural evolution of electrodes in lithium-ion batteries and how different chemistries in the cathode evolve during charge/discharge. The CGR and NCR cathodes adopt similar structures and $c$-lattice parameter evolution during most of charging, with the gradual expansion experienced ceasing near the charged state for both electrodes. The CGR electrode stabilizes and shows little change for the reminder of charge and during the potentiostatic step, whereas the NCR electrode contracts. This suggests that the extent of the $\mathrm{Li} / \mathrm{Ni}$ cation mixing, the $\mathrm{Ni}$ and $\mathrm{Li}$ contents of the electrode, and the presence of $\mathrm{Al}$ or $\mathrm{Mn}$ influence the cathode lithium extraction mechanism in differing ways near the charged state and subsequent discharge behavior. Decoupling the influence of each aspect may allow the design of better cathodes.

\section{ACKNOWLEDGEMENT}

Dr. Sharma acknowledges AINSE Ltd for providing support through the research fellowship scheme.

Dolotko, O., Senyshyn, A., Muhlbauer, M. J., Nikolowski, K., and Ehrenberg, H. (2014). "Understanding structural changes in NMC Li-ion cells by in situ neutron diffraction," J. Power Sources 255, 197-203.

Goodenough, J. B. and Kim, Y. (2011). "Challenges for rechargeable batteries," J. Power Sources 196, 6688-6694.

Hu, C.-W., Sharma, N., Chiang, C.-Y., Su, H.-C., Peterson, V. K., Hsieh, H.-W., Lin, Y.-F., Chou, W.-C., Shew, B.-Y., and Lee, C.-H. (2013). "Real-time investigation of the structural evolution of electrodes in a commercial lithium-ion battery containing a $\mathrm{V}$-added $\mathrm{LiFePO}_{4}$ cathode using in-situ neutron powder diffraction," J. Power Sources 244, 158-163.

Larson, A. C. and Von Dreele, R. B. (1994). General Structure Analysis System (GSAS) (Los Alamos National Laboratory Report LAUR 86-748).

Mohanty, D., Kalnaus, S., Meisner, R. A., Safat, A. S., Li, J., Payzant, A., Rhodes, K., Wood, D. L., and Daniel, C. (2013). "Structural transformation in a $\mathrm{Li}_{1.2} \mathrm{Co}_{0.1} \mathrm{Mn}_{0.55} \mathrm{Ni}_{0.15} \mathrm{O}_{2}$ lithium-ion battery cathode during high-voltage hold," RSC Adv. 3, 7479-7485.

Nagaura, T. and Tozawa, K. (1990). "Lithium ion rechargeable battery," Prog. Batteries Sol. Cells 9, 209.

Padhi, A. K., Nanjundaswamy, K. S., and Goodenough, J. B. (1997). "Phospho-olivines as positive-electrode materials for rechargeable lithium batteries," J. Electrochem. Soc. 144, 1188-1194.

Pang, W. K., Sharma, N., Peterson, V. K., Shiu, J.-J., and Wu, S. H. (2014). "In-situ neutron diffraction study of the simultaneous structural evolution of a $\mathrm{LiNi}_{0.5} \mathrm{Mn}_{1.5} \mathrm{O}_{4}$ cathode and $\mathrm{Li}_{4} \mathrm{Ti}_{5} \mathrm{O}_{12}$ anode in a $\mathrm{LiNi}_{0.5} \mathrm{Mn}_{1.5} \mathrm{Ollii}_{4} \mathrm{Ti}_{5} \mathrm{O}_{12}$ full cell," J. Power Sources 246, 464-472.

Richard, D., Ferrand, M., and Kearley, G. J. (1996). "Large array manipulation program," J. Neutron Res. 4, 33-39. 
Rodriguez, M. A., Ingersoll, D., Vogel, S. C., and Williams, D. J. (2004). "Simultaneous in siu neutron diffraction studies of the anode and cathode in lithium-ion cell," Electrochem. Solid-State Lett. 7, A8-A10.

Senyshyn, A., Muhlbauer, M. J., Nikolowski, K., Pirling, T., and Ehrenberg, H. (2012). "“In-operando" neutron scattering studies on Li-ion batteries," J. Power Sources 203, 126-129.

Sharma, N. and Peterson, V. K. (2012). "In situ neutron powder diffraction studies of lithium-ion batteries," J. Solid State Electrochem. 16, 1849-1856.

Sharma, N. and Peterson, V. K. (2013a). "Current dependency of lattice fluctuations and phase evolution of electrodes in lithium-ion batteries investigated by in situ neutron diffraction," Electrochim. Acta 101, 79-85.

Sharma, N. and Peterson, V. K. (2013b). "Overcharging a lithium-ion battery: effect on the $\mathrm{Li}_{x} \mathrm{C}_{6}$ negative electrode determined by in situ neutron diffraction," J. Power Sources 244, 695-701.

Sharma, N., Peterson, V. K., Elcombe, M. M., Avdeev, M., Studer, A. J., Blagojevic, N., Yusoff, R., and Kamarulzaman, N. (2010). "Structural changes in a commercial lithium ion battery during electrochemical cycling: an in-situ neutron diffraction study," J. Power Sources 195, 8258-8266.

Sharma, N., Du, G., Studer, A. J., Guo, Z., and Peterson, V. K. (2011a). "Structural change in the $\mathrm{MoS}_{2}$ anode of a $\mathrm{Li}$ ion battery during discharge: in-situ neutron diffraction studies using an optimised cell design," Solid State Ion. 199-200, 37-43.

Sharma, N., Reddy, M. V., Du, G., Adams, S., Chowdari, B. V. R., Guo, Z., and Peterson, V. K. (2011b). "Time-dependent in-situ neutron diffraction investigation of a $\mathrm{Li}\left(\mathrm{Co}_{0.16} \mathrm{Mn}_{1.84}\right) \mathrm{O}_{4}$ cathode," J. Phys. Chem. C 115, 21473-21480.

Sharma, N., Guo, X., Du, G., Guo, Z., Wang, J., Wang, Z., and Peterson, V. K. (2012). "Direct evidence of concurrent solid-solution and two-phase reactions and the nonequilibrium structural evolution of $\mathrm{LiFePO}_{4}$," J. Am. Chem. Soc. 134, 7867-7873.

Sharma, N., Yu, D., Zhu, Y., Wu, Y., and Peterson, V. K. (2013). "Non-equilibrium structural evolution of the lithium-rich $\mathrm{Li}_{1+\mathrm{y}} \mathrm{Mn}_{2} \mathrm{O}_{4}$ cathode within a battery," Chem. Mater. 25, 754-760.

Studer, A. J., Hagen, M. E., and Noakes, T. J. (2006). "Wombat: the high intensity powder diffractometer at the OPAL reactor," Physica B 385-386, 1013-1015.

Tarascon, J.-M. and Armand, M. (2001). "Issues and challenges facing rechargeable lithium batteries," Nature 414, 359-367.

Thackeray, M. M., de Kock, A., Rossouw, M. H., Liles, D., Bittihn, R., and Hoge, D. (1992). "Spinel electrodes from the Li-Mn-O system for rechargeable lithium battery applications," J. Electrochem. Soc. 139, 363-366.

Toby, B. H. (2001). "EXPGUI, a graphical user interface for GSAS," J. Appl. Crystallogr. 34, 210-213.

Wang, X.-L., An, K., Cai, L., Feng, Z., Nagler, S. E., Daniel, C., Rhodes, K. J., Stoica, A. D., Skorpenske, H. D., Liang, C., Zhang, W., Kim, J., Qi, Y., and Harris, S. J. (2012). "Visualizing the chemistry and structure dynamics in lithium-ion batteries by in-situ neutron diffraction," Sci. Rep. 2, 1-7. 